\title{
Methicillin-sensitive Staphylococcus aureus bacteremia in aged patients: the importance of formal infectious specialist consultation
}

\author{
E. Forsblom ${ }^{1}\left[\right.$ - A. Kakriainen ${ }^{1} \cdot$ E. Ruotsalainen ${ }^{1} \cdot$ A. Järvinen ${ }^{1}$
}

Received: 11 January 2018 / Accepted: 21 February 2018 / Published online: 6 March 2018

(c) The Author(s) 2018, corrected publication April 2018

\begin{abstract}
Background Infectious specialist consultations (ISC) provide ever more evidence for improved outcome in Staphylococcus aureus bacteremia (SAB). Most ISC are formal (bedside). However, the impact of ISC on clinical management and prognosis lacks evaluation in aged patients with SAB.

Methods Multicenter retrospective analysis of methicillin-sensitive (MS) SAB. Patients were stratified according to age $\geq 60$ years (sub-analyses for $\geq 75$ years and females) and formal (bedside) ISC given within 7 days of SAB diagnosis. The impact on management and outcome of formal ISC was explored. Statistics were performed with univariate analysis, Cox proportional hazards regression model analysis, including propensity-score adjustment, and graphic Kaplan-Meier interpretation.

Results Altogether 617 patients were identified and 520 (84\%) had formal ISC. Presence of formal ISC resulted in equivalent clinical management regardless of age over or under 60 years: localization and eradication of infection foci ( $80 \mathrm{vs.} 82 \%$ and 34 vs. $36 \%$ ) and use of anti-staphylococcal antibiotics (65 vs. 61\%). Patients aged $\geq 60$ years managed without formal ISC, compared to those with formal ISC, had less infection foci diagnosed (53 vs. 80\%, $p<0.001)$. Lack of formal ISC in patients aged $\geq 60$ years resulted in no infection eradication and absence of first-line anti-staphylococcal antibiotics. Formal ISC, compared to absence of formal ISC, lowered mortality at 90 days in patients aged $\geq 60$ years (24 vs. $47 \%, p=0.004)$. In Cox proportional regression, before and after propensity-score adjustment, formal ISC was a strong positive prognostic parameter in patients aged $\geq 60$ years (HR $0.45 ; p=0.004$ and HR $0.44 ; p=0.021$ ), in patients aged $\geq 75$ years (HR 0.18 ; $p=0.001$ and HR $0.11 ; p=0.003$ ) and in female patients aged $\geq 75$ years (HR $0.13 ; p=0.005$ ).

Conclusion Formal ISC ensures proper active clinical management irrespective of age and improve prognosis in aged patients with MS-SAB.
\end{abstract}

Keywords S. aureus bacteremia $\cdot$ High age $\cdot$ Infectious specialist consultation $\cdot$ Deep infection

\section{Introduction}

Staphylococcus aureus causes severe community- and healthcare-acquired bacteremia (SAB) [1,2]. Despite antistaphylococcal agents, new potent antimicrobials, and identification and eradication of infection foci [3, 4], the mortality in SAB remains high and ranges up to $30 \%$ [5]. Consultation by infectious specialist (ISC) provides evidence for

E. Forsblom

erik.forsblom@helsinki.fi

1 Division of Infectious Diseases, Inflammation Center, University of Helsinki and Helsinki University Central Hospital, Aurora Hospital, Nordenskiöldinkatu 26, Building 5, P.O. Box 348, 00029 HUS Helsinki, Finland improved clinical management of SAB. ISC enhances choice and duration of antibiotic treatment [6] and accelerate radiological diagnostics [7], localization [8] and eradication [9] of infection foci. Most ISC are given formal (bedside) and the superiority of formal ISC over other forms, e.g. informal (telephone) ISC, has been demonstrated [10]. However, most important, ISC improve outcome of SAB [7-10].

There is a rapid expansion of the geriatric population in the Western countries [11] with rising incidences of SAB among older persons $[12,13]$. Specific characteristics have been identified: aged SAB patients are comorbid, bacteremia often hospital-acquired and the mortality high $[14,15]$. However, few studies have compared clinical management and prognosis of aged versus young SAB patients and in many reports parameters that may influence prognosis, e.g. 
essential diagnostics, are unreported or performed rarely and deep infection foci documented scarcely or not identified [16-19]. The presence of ISC has been recorded in only three studies on aged patients with SAB reporting that patients were managed in conjunction with an ISC or that an ISC assisted in evaluating the source of SAB $[14,18]$, whereas one report concluded that aged patients were less likely to receive ISC guidance [19]. However, despite certification that ISC improve prognosis, the nature of ISC and the impact of ISC on clinical management and outcome have been neglected in previous reports on aged patients with SAB [14-19].

The objective was to explore how formal ISC impacts clinical management and prognosis of aged patients with methicillin-sensitive (MS) SAB. Main analyses were performed with patients aged $\geq 60$ years and sub-analyses with patients aged $\geq 75$ years and aged female patients. The inclusion of MS-SAB only enabled a setting where each patient received appropriate antimicrobial therapy and thus differences in empirical antibiotic therapy could be avoided.

\section{Materials and methods}

\section{Study population}

Multicenter and retrospective study with 90 days follow-up was performed. All adult patients with at least one blood culture for MS-SAB from five universities and seven central hospitals in Finland during January 1999-May 1999 and January 2000-August 2002 were recruited. Furthermore, as an extension, each adult patient with positive blood culture for $S$. aureus from Helsinki University Hospital in Finland in 2006-2007 was included. Two time-periods were mandatory to account for possible temporary differences in treatment management. Documentation included: gender, age, diseases, infection acquisition, illness severity, antibiotic therapy, infection foci, ISC, outcome and autopsy results. The primary outcome was mortality at 90 days. Secondary outcome measures were identification and eradication of infection foci and time to defervescence. Exclusions were: age $<18$ years, pregnancy, epilepsy, bacteremia 28 days prior to the study and poly-microbial bacteremia.

\section{Definitions}

Healthcare-acquired SAB was defined as bacteremia with the first positive blood culture for $S$. aureus received (1) $\geq 48 \mathrm{~h}$ after hospital admission or (2) within $48 \mathrm{~h}$ of hospital admission with a preceding previous hospital discharge within 1 week. Diseases were classified with McCabe's criteria [20]. Severe sepsis was categorized as sepsis in combination with hypotension, hypo-perfusion or organ failure
[21]. Formal ISC was defined as bedside consultation within 1 week of SAB diagnosis by infectious specialist including: review of patient records and physical investigation with comprehensive written directives into patient records on clinical management.

\section{Antibiotic therapy}

The standard antibiotic therapy was anti-staphylococcal penicillin cloxacillin. Patients with penicillin contradictions received cephalosporin, either cefuroxime of ceftriaxone, as a first-line alternative therapy, or vancomycin, clindamycin or a carbapenem as second-line choice. Rifampicin and/ or fluoroquinolone were provided as an adjunctive therapy. Antibiotic therapy was regarded correct when given intravenously for at least 28 days for a deep infection focus and 14 days in the absence of deep infection. Detailed information on indications, dosages and administration routes for antibiotic therapy has been provided in previous studies [22].

\section{Statistical analyses}

Categorical variables were compared with Pearson's $X^{2}$ test and non-categorical variables with Student's t-test. Odds ratios (OR) and hazard ratios (HR) with 95\% confidence intervals (CI) were calculated. Univariate factors with $p<0.1$ were entered into Cox regression model (proportional hazards regression). Furthermore, a propensity-score adjusted Cox regression model analysis was performed to evaluate the explicit prognostic impact of formal ISC in aged patients. First, propensity-scores were estimated by logistic regression and variables with significant connection to ISC identified. Second, a propensity-score adjusted Cox regression analysis was performed for prognostic parameters of 90 -day outcome. Tests were two-tailed and $p<0.05$ considered significant. Analyses were done with SPSS 12.0 (SPSS Inc., Chicago, IL, USA).

\section{Results}

\section{Patient characteristics}

A total of 617 SAB patients were identified. Altogether $46 \%$ (286) were aged $\geq 60$ years. Formal ISC was provided to $87 \%$ (250) of patients aged $\geq 60$ years and $82 \%$ (270) of patients aged $<60$ years (Fig. 1). When comparing patients aged $\geq 60$ years according to presence of formal ISC, no differences were seen regarding gender, SAB acquisition, previous hospitalization or underlying diseases. However, among patients receiving formal ISC, those aged $\geq 60$ years, compared to those $<60$ years, had more healthcare-acquired SAB (63 vs. $44 \%$ ), more previous hospitalization (62 vs. 
Fig. 1 Study profile. Presentation of 617 methicillinsensitive Staphylococcus aureus bacteremia patients stratified according to break-point age of 60 years and presence of formal (bedside) infectious specialist consultation (ISC) into different groups (groups 1-4)

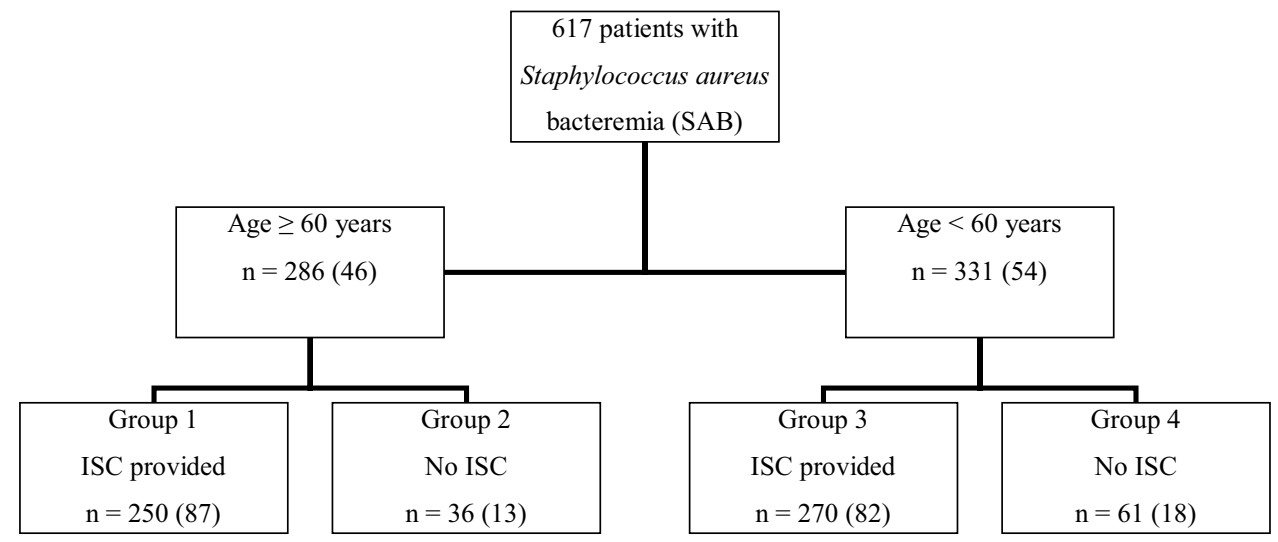

$42 \%)$ and less healthy or non-fatal underlying diseases (61 vs. $85 \%)(p<0.001)$. A total of $9 \%(56)$ had severe sepsis at blood culture collection. No differences in severe sepsis were observed for patients aged over or under 60 years when compared according to presence of formal ISC (Table 1).

\section{Deep infection foci}

Deep infection foci were identified in 75\% (463) of patients. In patients aged $\geq 60$ years, presence of formal ISC, compared to lack of formal ISC, resulted in more deep infection foci ( $80 \mathrm{vs.} 53 \%, p<0.001$ ), osteomyelitis or septic arthritis (38 vs. 8\%) and deep abscesses (34 vs. 6\%) $(p=0.001)$ localized. However, when comparing formal ISC for patients aged $\geq 60$ years with those aged $<60$ years, no difference was seen in the presence of deep infection foci ( 80 vs. $82 \%$ ) or peripheral or central venous catheters (18 vs. 12\%), whereas deep abscesses were encountered less (34 vs. 53\%, $p=0.001)$ and foreign body infections more (24 vs. $10 \%$, $p=0.001)$ (Table 1).

\section{Clinical management}

Trans-thoracic and trans-esophageal echocardiography (TTE and TEE) were provided to 64\% (183) and 20\% (57) of patients aged $\geq 60$ years. No difference in access to TTE was seen according to age over or under 60 years or presence or absence of formal ISC. However, in patients aged $\geq 60$ years formal ISC, compared to lack of formal ISC, resulted in more TEE (22 vs. $6 \%, p=0.021$ ). Formal ISC resulted in active infection eradication in patients aged $\geq 60$ years including infection removal (34\%), heart valve replacement $(<1 \%)$ and joint lavage $(3 \%)$. Formal ISC gave access to infection foci removal irrespective of age over or under 60 years. However, infection eradication was not provided to patients aged $\geq 60$ years managed without formal ISC (Table 1).

\section{Antibiotic therapy}

All patients were provided with an intravenous standard antimicrobial agent effective in vitro against $S$. aureus blood isolate from the first day of the positive blood culture: 53\% (327) received standard antibiotic cloxacillin, 37\% (228) cefuroxime or ceftriaxone and $2 \%$ (12) vancomycin. The remaining received clindamycin or a carbapenem. Adjunctive rifampicin and fluoroquinolone therapy was provided to altogether 57 and $49 \%$ of patients aged $\geq 60$ years, respectively. The total intravenous antibiotic duration for patients aged $\geq 60$ years receiving formal ISC was $24.6 \pm 13$ (days $\pm \mathrm{SD}$ ) and for patients managed without formal ISC $19.8 \pm 15$ (days \pm SD) $(p=0.038)$. Formal ISC resulted in equal treatment with cloxacillin, cephalosporin and adjunctive antibiotics fluoroquinolone or rifampicin for patients aged over or under 60 years. None of patients aged $\geq 60$ years that were managed without formal ISC received cloxacillin as first-line antibiotics. Moreover, administration of cephalosporins or other antibiotics as first-line choice was less common among patients aged $\geq 60$ receiving formal ISC compared to those managed without formal ISC (28 vs. $75 \%$ and 7 vs. $25 \%, p<0.001$ ) (Table 1 ).

\section{Time to defervescence}

Data for time to defervescence was retrieved for 578 patients. Patients aged $\geq 60$ years, compared to patients aged $<60$ years, had shorter mean time to defervescence (days $\pm \mathrm{SD})(5.1 \pm 7.7$ vs. $7.2 \pm 10.1)(p=0.006)$ (Fig. 2$)$. The impact of clinical management on defervescence in aged patients was explored by categorizing time to defervescence according to cut-off value of 7 days. However, formal ISC and localization or eradication of infection did not affect fever time in patients aged $\geq 60$ years (Table 2 ). 
Table 1 Patient demographics, severity of illness, clinical management and outcome in 617 methicillin-sensitive Staphylococcus aureus bacteremia patients stratified according to break-point age of 60 years and presence of formal (bedside) infectious specialist consultation (ISC)

\begin{tabular}{|c|c|c|c|c|c|c|c|}
\hline \multirow[t]{2}{*}{ Patient characteristics } & \multirow{2}{*}{$\begin{array}{l}\text { Group } 1 \\
\geq 60 \text { years } \\
\text { Formal ISC present } \\
n=250\end{array}$} & \multirow{2}{*}{$\begin{array}{l}\text { Group } 2 \\
\geq 60 \text { years } \\
\text { Formal ISC absent } \\
n=36\end{array}$} & \multirow{2}{*}{$\begin{array}{l}\text { Group } 3 \\
<60 \text { years } \\
\text { Formal ISC present } \\
n=270\end{array}$} & \multicolumn{2}{|c|}{ Group 1 vs. group 2} & \multicolumn{2}{|c|}{ Group 1 vs. group 3} \\
\hline & & & & OR $(95 \% \mathrm{CI})$ & $p$ value & OR $(95 \% \mathrm{CI})$ & $p$ value \\
\hline \multicolumn{8}{|l|}{ Demographics } \\
\hline Male sex & $144(58)$ & $23(64)$ & $182(67)$ & $0.77(0.37-1.59)$ & 0.47 & $0.65(0.46-0.94)$ & 0.21 \\
\hline Age, years ${ }^{\mathrm{a}}$ & $72.6 \pm 7.6$ & $71.1 \pm 7.9$ & $43.0 \pm 12.3$ & - & 0.29 & - & $<0.001$ \\
\hline Healthcare-acquired & $157(63)$ & $19(53)$ & $118(44)$ & $1.51(0.75-3.05)$ & 0.25 & $2.18(1.53-3.09)$ & $<0.001$ \\
\hline $\begin{array}{l}\text { Previous } \\
\text { hospitalization }^{\mathrm{b}}\end{array}$ & $154(62)$ & $25(69)$ & $114(42)$ & $0.71(0.33-1.50)$ & 0.36 & $2.19(1.55-3.12)$ & $<0.001$ \\
\hline Healthy-nonfatal $^{c}$ & $153(61)$ & $19(53)$ & $230(85)$ & $1.41(0.69-2.85)$ & 0.34 & $0.27(0.18-0.42)$ & $<0.001$ \\
\hline Severe sepsis ${ }^{\mathrm{d}}$ & $21(8)$ & $5(14)$ & $18(7)$ & $0.57(0.20-1.62)$ & 0.28 & $1.28(0.67-2.47)$ & 0.45 \\
\hline \multicolumn{8}{|l|}{ Infection foci } \\
\hline Deep infections & $200(80)$ & $19(53)$ & $222(82)$ & $3.58(1.74-7.38)$ & $<0.001$ & $0.87(0.56-1.34)$ & 0.52 \\
\hline Pneumonia & $99(40)$ & $12(33)$ & $103(38)$ & $1.31(0.63-2.74)$ & 0.47 & $1.06(0.75-1.51)$ & 0.73 \\
\hline Endocarditis & $39(16)$ & $2(6)$ & $47(17)$ & $3.14(0.73-13.6)$ & 0.11 & $0.88(0.55-1.39)$ & 0.58 \\
\hline Osteomyelitis $^{\mathrm{e}}$ & $94(38)$ & $3(8)$ & $110(41)$ & $6.63(1.98-22.2)$ & 0.001 & $0.87(0.61-1.24)$ & 0.44 \\
\hline Deep abscesses & $85(34)$ & $2(6)$ & $143(53)$ & $8.76(2.05-37.3)$ & 0.001 & $0.46(0.32-0.65)$ & 0.001 \\
\hline $\begin{array}{l}\text { Any foreign body } \\
\text { infection }\end{array}$ & $59(24)$ & $4(11)$ & $27(10)$ & $2.47(0.84-7.27)$ & 0.09 & $2.78(1.69-4.55)$ & 0.001 \\
\hline Infected PVC or CVC & $45(18)$ & 0 & $32(12)$ & - & - & $1.48(0.89-2.44)$ & 0.12 \\
\hline \multicolumn{8}{|l|}{ Clinical management } \\
\hline $\begin{array}{l}\text { TTE } \\
\text { echocardiography }\end{array}$ & $165(66)$ & $19(53)$ & $174(64)$ & $1.74(0.86-3.51)$ & 0.12 & $1.07(0.75-1.54)$ & 0.71 \\
\hline $\begin{array}{l}\text { TEE } \\
\text { echocardiography }\end{array}$ & $55(22)$ & $2(6)$ & $54(21)$ & $4.79(1.12-20.6)$ & 0.021 & $1.13(0.74-1.72)$ & 0.58 \\
\hline $\begin{array}{l}\text { Any infection foci } \\
\text { removal }\end{array}$ & $84(34)$ & 0 & $94(36)$ & - & - & $0.95(0.66-1.36)$ & 0.77 \\
\hline $\begin{array}{l}\text { Heart valve replace- } \\
\text { ment }\end{array}$ & $2(<1)$ & 0 & $4(1)$ & - & - & $0.54(0.09-2.95)$ & 0.47 \\
\hline Infected joint lavage & $7(3)$ & 0 & $6(2)$ & - & - & $1.27(0.42-3.82)$ & 0.67 \\
\hline \multicolumn{8}{|l|}{ Standard antibiotics } \\
\hline Cloxacillin & $163(65)$ & 0 & $165(61)$ & - & - & $1.19(0.83-1.70)$ & 0.33 \\
\hline Cephalosporin & $70(28)$ & $27(75)$ & $85(31)$ & $0.13(0.06-0.29)$ & $<0.001$ & $0.85(0.58-1.23)$ & 0.39 \\
\hline Other antibiotics & $17(7)$ & $9(25)$ & $20(7)$ & $4.57(1.87-11.2)$ & $<0.001$ & $1.09(0.56-2.14)$ & 0.79 \\
\hline Treatment duration ${ }^{\mathrm{h}}$ & $24.6 \pm 13$ & $19.8 \pm 15$ & $26.1 \pm 12$ & - & 0.038 & - & 0.19 \\
\hline \multicolumn{8}{|l|}{ Adjunctive antibiotics } \\
\hline Fluoroquinolone & $129(52)$ & $11(31)$ & $136(50)$ & $2.42(1.14-5.14)$ & 0.018 & $1.05(0.75-1.48)$ & 0.78 \\
\hline Rifampicin & $152(61)$ & $11(31)$ & $175(65)$ & $3.53(1.66-7.49)$ & 0.001 & $0.84(0.59-1.20)$ & 0.34 \\
\hline \multicolumn{8}{|l|}{ Outcome } \\
\hline Hospital duration ${ }^{\mathrm{h}}$ & $38.7 \pm 34$ & $31.6 \pm 26$ & $32.7 \pm 29$ & - & 0.09 & - & 0.10 \\
\hline Mortality in 28 days & $46(18)$ & $11(31)$ & $12(4)$ & $0.51(0.24-1.12)$ & 0.088 & $4.85(2.50-9.39)$ & $<0.001$ \\
\hline Mortality in 90 days & $61(24)$ & $17(47)$ & $21(8)$ & $0.36(0.18-0.74)$ & 0.004 & $3.83(2.25-6.51)$ & $<0.001$ \\
\hline
\end{tabular}

Altogether $61(18 \%)$ patients were aged $<60$ years and received no formal ISC and are omitted in the presentation. Values are expressed as number of patients (\%), odds ratios (ORs) with $95 \%$ confidence intervals (CI) and mean \pm standard deviation (SD)

${ }^{\text {a }}$ Student's $t$ test (mean $\left.\pm \mathrm{SD}\right)$

${ }^{\mathrm{b}}$ Within 2 months preceding SAB

${ }^{\mathrm{c}}$ McCabe's classification [20]

${ }^{\mathrm{d}}$ At blood culture collection

${ }^{\mathrm{e}}$ Including septic arthritis

${ }^{\mathrm{f}}$ Peripheral or central venous catheter

${ }^{g}$ Thoracic or -esophageal

${ }^{\mathrm{h}}$ Days $($ mean $\pm \mathrm{SD})$ 


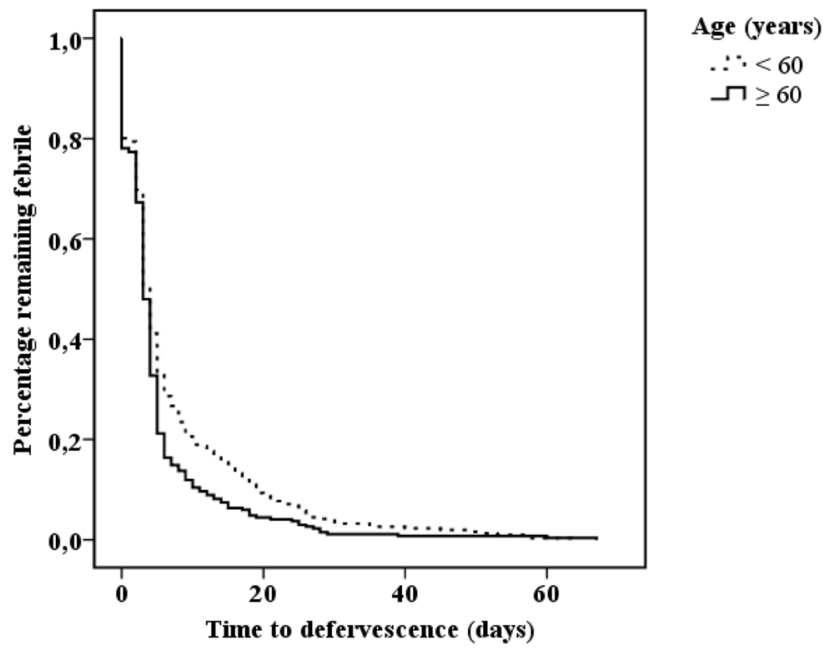

Fig. 2 Kaplan-Meier analysis for time to defervescence (days) in 578 methicillin-sensitive Staphylococcus aureus bacteremia patients categorized according to the break-point age 60 years. $\log$ rank $=0.006$

\section{Outcome}

The total hospital duration for patients aged $\geq 60$ years receiving formal ISC was $38.7 \pm 34$ (days \pm SD) and for patients managed without formal ISC $31.6 \pm 26$ (days \pm SD) ( $p$ value $=0.09$ ). The overall case fatality in 617 patients was $13 \%$ at 28 days and $19 \%$ at 90 days. Patients aged $\geq 60$ years receiving formal ISC, compared to those without, had lower mortality at 28 days ( 18 vs. $31 \%, p=0.088)$ and at 90 days ( 24 vs. $47 \%, p=0.004$ ) (Table 1 ). Parameters for 90-days mortality in patients aged $\geq 60$ years were evaluated (Table $3 \mathrm{a}, \mathrm{b}$ ). In univariate analysis, factors with positive prognosis were lack of underlying diseases (OR 0.27, $p<0.001$ ), formal ISC (OR 0.36, $p=0.004$ ) and adjunctive rifampicin therapy (OR $0.31, p<0.001)$, whereas poor prognosis connected to severe sepsis (OR 2.52, $p=0.023$ ), endocarditis (OR 2.41, $p=0.01$ ) and pneumonia (OR 3.98, $p<0.001$ ) (Table 3a). In Cox proportional regression analysis lack of underlying diseases (HR $0.38, p<0.001$ ), formal ISC (HR $0.45, p=0.004$ ) and adjunctive rifampicin therapy (HR $0.32, p<0.001$ ) were positive prognostic parameters, whereas severe sepsis (HR 1.98, $p=0.039$ ), endocarditis (HR 2.09, $p=0.01$ ) and pneumonia (HR 2.58, $p<0.001$ ) were associated to poor prognosis (Table 3). When Cox proportional regression was repeated by including only patients aged $\geq 75$ years or including only female patients aged $\geq 75$ years, the positive prognostic impact of formal ISC remained (HR 0.18, $p=0.001$ and HR 0.13, $p=0.005$ ).

In propensity-score-adjusted Cox proportional analysis, parameters for 90-days outcome were: formal ISC (HR 0.44, $p=0.021$ ), lack of underlying diseases (HR $0.37, p<0.001$ ), severe sepsis (HR 1.99, $p=0.039$ ), endocarditis (HR 2.06, $p=0.014$ ), pneumonia (HR 2.57, $p<0.001$ ) and rifampicin adjunctive therapy (HR $0.31, p<0.001$ ) (Table 4$)$. When propensity-score-adjusted Cox proportional regression was repeated by including only patients aged $\geq 75$ years or including only female patients aged $\geq 75$ years, the impact of formal ISC remained (HR 0.11, $p=0.003$ and HR 0.13, $p=0.005)$.

To further evaluate differences between patients aged over or under 60 years, autopsy results were examined for altogether 57 patients. The immediate causes of death were stratified according to age. For patients aged $<60$ years $(n=15)$, the three most common immediate causes of
Table 2 The impact of clinical management on time to defervescence stratified according to cut-off value of 7 days in 268 methicillinsensitive Staphylococcus aureus bacteremia patients aged $\geq 60$ years

\begin{tabular}{lclll}
\hline Clinical management & \multicolumn{2}{l}{ Time to defervescence } & \\
\cline { 2 - 4 } & $\begin{array}{l}<7 \text { days } \\
n=227(85)\end{array}$ & $\begin{array}{l}\geq 7 \text { days } \\
n=41(15)\end{array}$ & OR (95\% CI) & $p$ value \\
\hline Formal ISC & $209(92)$ & $35(85)$ & $1.99(0.74-5.36)$ & 0.17 \\
Deep infection & $173(76)$ & $35(85)$ & $0.55(0.22-1.38)$ & 0.19 \\
Endocarditis & $34(15)$ & $7(17)$ & $0.86(0.35-2.09)$ & 0.73 \\
Pneumonia & $86(38)$ & $19(46)$ & $0.71(0.36-1.38)$ & 0.31 \\
Osteomyelitis & $81(36)$ & $14(34)$ & $1.07(0.53-2.16)$ & 0.85 \\
Foreign body infection & $52(23)$ & $8(20)$ & $1.23(0.53-2.82)$ & 0.63 \\
Surgical-radiological infection & $74(33)$ & $10(24)$ & $1.49(0.69-3.22)$ & 0.29 \\
$\quad$ removal & & & & - \\
Heart valve replacement & $2(1)$ & 0 & - & - \\
Infected joint lavage & $7(3)$ & 0 & - & \\
\hline
\end{tabular}

Values are expressed as number of patients (\%) and odds ratios (ORs) with 95\% confidence intervals (CI) or mean \pm standard deviation according to Student's $t$ test

${ }^{a}$ Including septic arthritis 
Table 3 Cox proportional regression for prognostic factors of 90-day mortality in methicillin-sensitive Staphylococcus aureus bacteremia patients aged $\geq 60$ years $(n=286)$

\begin{tabular}{|c|c|c|c|c|c|c|}
\hline \multirow[t]{2}{*}{ Patient characteristics } & \multicolumn{2}{|l|}{ Outcome } & \multicolumn{2}{|l|}{ Univariate analysis } & \multicolumn{2}{|l|}{ Cox regression } \\
\hline & $\begin{array}{l}\text { Died } \\
n=78(27)\end{array}$ & $\begin{array}{l}\text { Survived } \\
n=208(73)\end{array}$ & OR $(95 \%$ CI $)$ & $p$ value & HR (95\% CI) & $p$ value \\
\hline Male sex & $46(59)$ & $121(58)$ & $1.03(0.61-1.75)$ & NS & - & - \\
\hline Healthcare-acquired & $48(62)$ & $128(62)$ & $1.00(0.59-1.71)$ & NS & - & - \\
\hline Healthy—nonfatal ${ }^{\mathrm{a}}$ & $29(37)$ & $143(69)$ & $0.27(0.16-0.46)$ & $<0.001$ & $0.38(0.24-0.61)$ & $<0.001$ \\
\hline Severe sepsis ${ }^{b}$ & $12(15)$ & $14(7)$ & $2.52(1.11-5.72)$ & 0.023 & $1.98(1.04-3.79)$ & 0.039 \\
\hline Formal ISC $^{\mathrm{c}}$ & $61(78)$ & $189(91)$ & $0.36(0.18-0.74)$ & 0.004 & $0.45(0.26-0.78)$ & 0.004 \\
\hline Endocarditis & $18(23)$ & $23(11)$ & $2.41(1.22-4.77)$ & 0.01 & $2.09(1.19-3.64)$ & 0.01 \\
\hline Pneumonia & $49(63)$ & $62(30)$ & $3.98(2.30-6.88)$ & $<0.001$ & $2.58(1.59-4.18)$ & $<0.001$ \\
\hline Rifampicin ${ }^{\mathrm{d}, \mathrm{e}}$ & $20(26)$ & $110(53)$ & $0.31(0.17-0.55)$ & $<0.001$ & $0.32(0.19-0.54)$ & $<0.001$ \\
\hline Fluoroquinolone $^{\mathrm{d}}$ & $35(45)$ & $105(50)$ & $0.79(0.47-1.35)$ & NS & - & - \\
\hline
\end{tabular}

Values are expressed as number of patients $(\%)$ and odds ratios (OR), hazards ratio (HR) and $95 \%$ confidence intervals (95\% CI) are presented

$N S$ non-significant

àcCabe's classification [20]

${ }^{\mathrm{b}}$ At blood culture collection time-point

${ }^{\mathrm{c}}$ Infectious specialist consultation

${ }^{\mathrm{d}}$ Adjunctive therapy

${ }^{\mathrm{e}}$ For at least 14 days
Table 4 Propensity-score-adjusted Cox proportional regression analysis for 90-day mortality in methicillin-sensitive Staphylococcus aureus bacteremia patients aged $\geq 60$ years $(n=286)$ according to infectious specialist consultation

\begin{tabular}{llr}
\hline Patient characteristics & $\begin{array}{l}\text { Propensity-score-adjusted mul- } \\
\text { tivariate analysis HR (95\% CI) }\end{array}$ & $p$ value \\
\hline Absence of formal ISC $^{\mathrm{a}}$ & 1.0 & - \\
Presence of formal ISC $^{\mathrm{a}}$ & $0.44(0.22-0.88)$ & 0.021 \\
Healthy-nonfatal $^{\mathrm{b}}$ & $0.37(0.23-0.60)$ & $<0.001$ \\
Severe sepsis $^{\mathrm{c}}$ & $1.99(1.03-3.81)$ & 0.039 \\
Endocarditis $^{\text {Pneumonia }}$ & $2.06(1.16-3.67)$ & 0.014 \\
Rifampicin $^{\mathrm{d}, \mathrm{e}}$ & $2.57(1.58-4.17)$ & $<0.001$ \\
\hline
\end{tabular}

${ }^{a}$ Infectious specialist consultation

${ }^{\mathrm{b}}$ McCabe's classification [20]

${ }^{\mathrm{c}}$ At blood culture collection time-point

${ }^{\mathrm{d}}$ Adjunctive therapy

${ }^{\mathrm{e}}$ For at least 14 days

death were acute subarachnoid or gastrointestinal hemorrhages $(43 \%)$, severe sepsis $(20 \%)$ or pneumonia $(20 \%)$. For patients aged $\geq 60$ years $(n=42)$, the corresponding causes were $\mathrm{SAB}$ with or without a background condition (43\%), acute cardio-pulmonary disease (21\%) or deep infection $(19 \%)$. Altogether, the immediate causes of death were infection related in $67 \%$ of patients aged $\geq 60$ years and $60 \%$ in patients aged $<60$ years.

\section{Discussion}

The main observations were that formal ISC ensures proper clinical management irrespective of age and formal ISC improves prognosis of aged patients with MSSAB. Accounting for all prognostic parameters, patients aged $\geq 60$ years, patients aged $\geq 75$ years and female patients aged $\geq 75$ years, had a more than twofold lower odds ratio for a fatal outcome due to formal ISC.

Previous reports connect ISC to improved antibiotic therapies, accelerated diagnostics and eradications of deep infection foci and better outcomes in SAB [6-10, 23]. Corresponding observations were seen in the present study. We provided formal ISC within 7 days of SAB diagnosis and, among patients aged $\geq 60$ years, this resulted in echocardiography in up to $66 \%$, deep infection foci identification in $80 \%$ and infection eradication provided to $34 \%$ of patients. Formal ISC resulted in radiological infection diagnostics, localization and eradication irrespective of age over or under 60 years. However, patients aged $\geq 60$ years managed without formal ISC had less infection foci diagnosed and were provided no infection foci eradications compared to those receiving formal ISC. Similar trends were observed also for antibiotics. Patients aged $\geq 60$ years managed by formal ISC had anti-staphylococcal penicillin as first-line alternative in $2 / 3$ of cases, whereas cephalosporins or other antibiotics were used in under $1 / 3$ and adjunctive antibiotics in over $50 \%$ of cases. Most important, formal ISC resulted in antibiotic therapies irrespective of age over or under 60 years. Contrary, no of patients aged $\geq 60$ years managed without 
formal ISC had anti-staphylococcal penicillin as first-line antibiotics, whereas $3 / 4$ had cephalosporins as first-line alternative and adjunctive antibiotics were provided in only $1 / 3$ of cases.

Deep infection foci have been identified in only $14-31 \%$ of aged patients in earlier studies on SAB [14-16, 18, 24]. Previous reports have stated that aged SAB patients are less likely to receive ISC, echocardiography or infection foci identification or eradication, and extensive diagnostics have not been pursued in aged patients due to presumed poor outcome $[17,19,24,25]$. To the best of our knowledge, only three studies report presence of ISC stating that patients were managed in conjunction with an ISC $[14,18]$, whereas one report concluded that aged patients were less likely to receive ISC guidance [19]. However, the content or nature of ISC or the impact of ISC on disease progression or prognosis was not evaluated [14, 18, 19]. The importance of infection identification and eradication in SAB has been confirmed $[4,26]$ and lack of echocardiography and undiagnosed infection foci have been suspected to connect to mortality in aged SAB patients $[14,17]$. Autopsy examinations in the present study revealed that for deceased patients both bacteremia and deep infection foci account for a larger part of mortalities of aged patients compared to young ones. The present study demonstrate that formal ISC-guided clinical management result in frequent localization and eradication of deep infection with subsequent positive prognostic impact in aged patients with SAB.

Comparison of prognosis in aged and young $\mathrm{SAB}$ patients is challenging due to differences in age categorizations and reported follow-up time and in earlier studies. We applied break-point ages of 60 and 75 years with mortality rates for patients aged over 60 years at 28 and 90 days of 18 and $24 \%$ for patients managed by formal ISC and 31 and $47 \%$ for patients not receiving formal ISC. Previous reports have presented results according to mean ages of 63-85 years $[14,15]$ or break-point age of 60-65 years $[16,18,19]$. In older patients, the mortalities have varied considerably with $15 \%$ at 7 days [15], $11-36 \%$ at 30 days [18, 24], 29\% at 90 days [17] and 33-56\% at 6 months [14]. The present study observed higher fatalities for aged patients compared to young. This is in line with earlier reports $[16,17$, $19,24]$ but deviate from one study with no link between age and clinical outcome [18]. Previous reports have presented age, comorbidity, MRSA and unknown bacteremia source as independent parameters for mortality among aged patients with SAB [14, 16, 17, 19, 24]. Parameters influencing outcome in patients aged $\geq 60$ years in the present study have been identified earlier in SAB patient cohorts without age specification, i.e. comorbidity $[5,6]$, severity of illness [7], ISC [7-10], pneumonia or endocarditis $[7,10]$ and rifampicin therapy [22]. Moreover, adjunctive fluoroquinolone therapy did not impact outcome and this is in line with previous observations [22].

Previous studies on aged SAB patients do not report fever [14-16, 18, 24], whereas two studies reported that aged $\mathrm{SAB}$ patients were more likely to be afebrile prior to $\mathrm{SAB}[17,19]$. In the present study, defervescence was significantly shorter for aged patients compared to young. We have previously shown that ISC reduced fever duration in SAB [10]. However, in the present study clinical management or formal ISC had no impact on defervescence in patients aged $\geq 60$ years. Hence, time to defervescence among patients aged $\geq 60$ years does not seem to correlate with treatment strategies.

MRSA weakens prognosis [27] and MRSA in previous reports on aged SAB patients has been as much as $52-100 \%$ [14-16, 18]. We included only MS-SAB. Thus, patients had proper antibiotic therapy from the first day of positive blood culture excluding bias from differences in empirical antibiotic therapy. In the present study $1 / 2$ of patients aged $\geq 60$ years received adjunctive rifampicin therapy. We have shown that rifampicin adjunctive therapy may impact prognosis positively $[22,28]$. However, among patients aged $\geq 60$ years, the risk of drug interactions may have resulted in a selection bias favoring rifampicin treatment in less comorbid patients and hence, the potential positive prognostic impact of rifampicin in aged patients should be interpreted with caution. The authors recommend that prospective randomized clinical trials evaluate the potential advantage of rifampicin therapy before it can be routinely recommended as part of treatment of aged patients with SAB.

There are weaknesses in the present study that have to be accounted for when interpreting results. First, the retrospective design includes risk for bias due to differences in patient groups. However, propensity-score adjustment may reduce potential bias [29]. Second, aged infectious patients may lack fever and present non-specific symptoms. Moreover, declined functional status has been connected to mortality among aged infectious patients [30]. Corresponding data were not recorded. Third, the patient cohort of the present study was originally collected to investigate trends of nosocomial SAB and prognostic impact of fluoroquinolones, rifampicin and ISC $[10,12,22,28]$. However, the authors noticed that, although the incidence of SAB among older persons is rising, the prognostic impact of ISC has not been extensively evaluated among aged patients with SAB. Regarding the earlier time-period of the patient cohort, the question of whether the patient data in the present study is valid to current clinical practice may be raised. Management of SAB is continuously developed, however, fundamental elements of SAB management remain unchanged over the years, e.g. the importance of proper non-delayed antibiotic therapy and diagnostics of infection foci. The authors view 
that the high presence of formal ISC has ensured recording of relevant data and high-standard management of SAB. Hence, the authors conclude that the patient data are not outdated for current clinical practice.

\section{Conclusion}

Formal ISC ensures proper and active clinical management irrespective of age and improve prognosis in aged patients with MS-SAB. The authors encourage clinicians to manage aged patients with MS-SAB through formal ISC guidance.

Funding The study has been supported by Grants from Helsinki University Central Hospital, the Medical Society of Finland and Svenska Kulturfonden. The funders had no role in study design, data collection and analysis, decision to publish, or preparation of the manuscript. The study was funded by Maud Kuistila Foundation and Perkléns Foundation.

\section{Compliance with ethical standards}

Conflict of interest The authors declare that they have no conflict of interest.

Ethics statement The trial was approved by The Institutional Review Board of Helsinki University Central Hospital and The Ethical Committee of Helsinki University Central Hospital.

Informed consent For this type of study formal consent is not required.

Open Access This article is distributed under the terms of the Creative Commons Attribution 4.0 International License (http://creativeco mmons.org/licenses/by/4.0/), which permits use, duplication, adaptation, distribution and reproduction in any medium or format, as long as you give appropriate credit to the original author(s) and the source, provide a link to the Creative Commons license and indicate if changes were made.

\section{References}

1. Jensen AG, Wachmann CH, Espersen F, Scheibel J, Skinhoj P, Frimodt-Moller N (2002) Treatment and outcome of Staphylococcus aureus bacteremia: a prospective study of 278 cases. Arch Intern Med 162:25-32

2. Chong YP, Park SJ, Kim HS, Kim ES, Kim MN, Park KH et al (2013) Persistent Staphylococcus aureus bacteremia: a prospective analysis of risk factors, outcomes, and microbiologic and genotypic characteristics of isolates. Medicine (Baltimore) 92:98-108

3. Thwaites GE, Edgeworth JD, Gkrania-Klotsas E, Kirby A, Tilley R, Török ME et al (2011) Clinical management of Staphylococcus aureus bacteraemia. Lancet Infect Dis 11:208-222

4. Jensen AG (2002) Importance of focus identification in the treatment of Staphylococcus aureus bacteraemia. J Hosp Infect 52:29-36

5. Kobayashi D, Yokota K, Takahashi O, Arioka H, Fukui T (2014) A predictive rule for mortality of inpatients with Staphylococcus aureus bacteraemia: a classification and regression tree analysis. Eur J Intern Med 25:914-918
6. Pragman AA, Kuskowski MA, Abraham JM, Filice GA (2012) Infectious disease consultation for Staphylococcus aureus bacteremia improves patient management and outcomes. Infect Dis Clin Pract 20:261-267

7. Rieg S, Peyerl-Hoffmann G, de With K (2009) Mortality of $S$. aureus bacteremia and infectious diseases specialist consultation-a study of 521 patients in Germany. J Infect 59:232-239

8. Robinson JO, Pozzi-Langhi S, Phillips M, Pearson JC, Christiansen KJ, Coombs GW et al (2012) Formal infectious diseases consultation is associated with decreased mortality in Staphylococcus aureus bacteraemia. Eur J Clin Microbiol Infect Dis 31:2421-2428

9. Lahey T, Shah R, Gittzus J, Schwartzman J, Kirkland K (2009) Infectious disease consultation lowers mortality from Staphylococcus aureus bacteremia. Medicine 88:263-267

10. Forsblom E, Ruotsalainen E, Ollgren J, Järvinen A (2012) Telephone consultation cannot replace bedside infectious disease consultation in the management of Staphylococcus aureus bacteraemia. Clin Infect Dis 56:527-535

11. National Center for Chronic Disease Prevention and Health Promotion DoPH, CDC (2013) The state of aging and health in America 2013

12. Lyytikäinen $\mathrm{O}$, Ruotsalainen $\mathrm{E}$, Järvinen $\mathrm{A}$, Valtonen $\mathrm{V}$, Ruutu P (2005) Trends and outcome of nosocomial and communityassociated bloodstream infections due to Staphylococcus aureus in Finland, 1995-2001. Eur J Clin Microbiol Infect Dis 24:399-404

13. Benfield T, Espersen F, Frimodt-Møller N, Jensen AG, Larsen AR, Pallesen LV et al (2007) Increasing incidence but decreasing in-hospital mortality of adult Staphylococcus aureus bacteraemia between 1981 and 2000. Clin Microbiol Infect 13:257-263

14. Big C, Malani PN (2010) Staphylococcus aureus bloodstream infections in older adults: clinical outcomes and risk factors for in-hospital mortality. J Am Geriatr Soc 58:300-305

15. Bader MS (2006) Staphylococcus aureus bacteremia in older adults: predictors of 7-day mortality and infection with a methicillin-resistant strain. Infect Control Hosp Epidemiol 27:1219-1225

16. Tacconelli E, Pop-Vicas AE, D'Agata EM (2006) Increased mortality among elderly patients with meticillin-resistant Staphylococcus aureus bacteraemia. J Hosp Infect 64:251-256

17. McClelland RS, Fowler VG Jr, Sanders LL, Gottlieb G, Kong LK, Sexton DJ et al (1999) Staphylococcus aureus bacteremia among elderly vs younger adult patients: comparison of clinical features and mortality. Arch Intern Med 14:1244-1247

18. Kullar R, Rybak MJ, Kaye KS (2013) Comparative epidemiology of bacteremia due to methicillin-resistant Staphylococcus aureus between older and younger adults: a propensity score analysis. Infect Control Hosp Epidemiol 34:400-406

19. Yahav D, Schlesinger A, Shaked H (2017) Clinical presentation, management and outcomes of Staphylococcus aureus bacteremia (SAB) in older adults. Aging Clin Exp Res 29:127-133

20. McCabe WR, Jackson GG (1962) Gram-negative bacteremia: I. Etiology and ecology. Arch Intern Med 110:847-855

21. Levy MM, Fink MP, Marshall JC, Abraham E, Angus D, Cook D et al (2003) 2001 SCCM/ESICM/ACP/ATS/SIS international sepsis definitions conference. Crit Care Med 31:1250-1256

22. Ruotsalainen E, Järvinen A, Koivula I, Kauma H, Rintala E, Lumio J et al (2006) Levofloxacin does not decrease mortality in Staphylococcus aureus bacteraemia when added to the standard treatment: a prospective and randomized clinical trial of 381 patients. J Intern Med 259:179-190

23. Vogel M, Schmitz RP, Hagel S, Pletz MW, Gagelmann N, Scherag A et al (2016) Infectious disease consultation for Staphylococcus aureus bacteremia-a systematic review and meta-analysis. J Infect 72:19-28

24. Kang CI, Song JH, Ko KS, Chung DR, Peck KR, Asian Network for Surveillance of Resistant Pathogens (ANSORP) Study Group 
(2011) Clinical features and outcome of Staphylococcus aureus infection in elderly versus younger adult patients. Int J Infect Dis 15:58-62

25. Yahav D, Eliakim-Raz N, Leibovici L, Paul M (2016) Bloodstream infections in older patients. Virulence 2:341-352

26. Kim SH, Park WB, Lee KD (2003) Outcome of Staphylococcus aureus bacteremia in patients with eradicable foci versus noneradicable foci. Clin Infect Dis 37:794-799

27. Cosgrove SE, Sakoulas G, Perencevich EN, Schwaber MJ, Karchmer AW, Carmeli Y (2003) Comparison of mortality associated with methicillin-resistant and methicillin-susceptible Staphylococcus aureus bacteremia: a meta-analysis. Clin Infect Dis 36:53-59
28. Forsblom E, Ruotsalainen E, Järvinen A (2015) Improved outcome with early rifampicin combination treatment in methicillinsensitive Staphylococcus aureus bacteraemia with a deep infection focus - a retrospective cohort study. PLoS One 10:e0122824

29. Rosenbaum PR, Rubin DB (1983) The central role of the propensity score in observational studies for causal effects. Biometrika 70:41-55

30. Deulofeu F, Cervello B, Capell S, Martí C, Mercadé V (1998) Predictors of mortality in patients with bacteremia: the importance of functional status. J Am Geriatr Soc 46:14-18 